\title{
CHOQUET INTEGRALS OF WEIGHTED TRIANGULAR FUZZY LINGUISTIC INFORMATION AND THEIR APPLICATIONS TO MULTIPLE ATTRIBUTE DECISION MAKING
}

\author{
Rui LIN ${ }^{1}$, Guiwu WEI ${ }^{2}$, Hongjun WANG ${ }^{3}$, Xiaofei ZHAO $^{4}$ \\ School of Economics and Management, Chongqing University \\ of Arts and Sciences, Chongqing 402160, P.R.China \\ E-mail: ${ }^{2}$ weiguiwu@163.com (corresponding author) \\ Received 16 December 2012; accepted 04 February 2013
}

\begin{abstract}
We investigate the multiple attribute decision making problems in which attribute values take the form of triangular fuzzy linguistic information. Firstly, the definition and some operational laws of triangular fuzzy linguistic are introduced. Then, we have developed three fuzzy linguistic Choquet integral aggregation operators: fuzzy linguistic choquet ordered averaging operator, fuzzy linguistic choquet ordered geometric operator and fuzzy linguistic choquet ordered harmonic mean operator. The prominent characteristic of the operators is that they cannot only consider the importance of the elements or their ordered positions, but also reflect the correlation among the elements or their ordered positions. We have studied some desirable properties of these operators, such as commutativity, idempotency and monotonicity, and applied these operators to multiple attribute decision making with triangular fuzzy linguistic information. Finally an illustrative example has been given to show the developed method.
\end{abstract}

Keywords: multiple attribute decision making (MADM), operational laws, triangular fuzzy linguistic variables, fuzzy linguistic choquet ordered averaging (FLCOA) operator, fuzzy linguistic choquet ordered geometric (FLCOG) operator, fuzzy linguistic choquet ordered harmonic mean (FLCOHM) operator.

JEL Classification: C61.

\section{Introduction}

Multiple attribute decision making (MADM) refers to making choice of the best alternative from among a finite set of decision alternatives in terms of multiple usually conflicting attributes (or called criteria) (Liu 2009a, 2009b; Zhang, Liu 2010a, 2010b; Liu, Su 2010; Liu, Zhang 2010, 2011; Liu et al. 2011a, 2011b; Liu, Wang 2011; Merigó, Gil-Lafuente 2009, 2010; Merigó, Casanovas 2009, 2010; Merigó 2010; Merigó et al. 2010; Tan, Chen 2010; Wang 2009a, 2009b, 2010; Wei 2008, 2009a-b, 2010a-d; Wei et al. 2010a, 2010b; Xu 2004a-c, 2005a-c, 2006a-d, 2007a-c, 2009b; 2010; Ye 2009a, $2009 \mathrm{~b})$. However, under many conditions, for the real multiple attribute decision making problems, the decision information about alternatives is usually uncertain or fuzzy due to the increasing complexity of the socio-economic environment and the vagueness 
of inherent subjective nature of human think, thus, numerical values are inadequate or insufficient to model real-life decision problems. Indeed, human judgments including preference information may be stated in triangular fuzzy linguistic variables. In order to effectively avoid the loss and distortion of information in triangular fuzzy linguistic information processing process, $\mathrm{Xu}(2007 \mathrm{~b})$ proposed the triangular fuzzy linguistic representation and computational model which has a distinct advantage over other linguistic processing methods in accuracy and reliability and developed some operators for aggregating triangular fuzzy linguistic variables, such as the fuzzy linguistic averaging (FLA) operator, fuzzy linguistic weighted averaging (FLWA) operator, fuzzy linguistic ordered weighted averaging (FLOWA) operator, and induced FLOWA (IFLOWA) operator. Zhao and Wei (2009) proposed some operators for aggregating triangular fuzzy linguistic variables, such as the fuzzy linguistic geometric averaging (FLGA) operator, fuzzy linguistic weighted geometric averaging (FLWGA) operator, fuzzy linguistic ordered weighted geometric (FLOWG) operator, and induced FLOWG (IFLOWG) operator and developed an approach based on FLWGA and IFLOWG operator to multiple attribute group decision making with triangular fuzzy linguistic variables. Wei (2009c) proposed some operators for aggregating triangular fuzzy linguistic variables, such as the fuzzy linguistic harmonic mean (FLHM) operator, fuzzy linguistic weighted harmonic mean (FLWHM) operator, fuzzy linguistic ordered weighted harmonic mean (FLOWHM) operator, and fuzzy linguistic hybrid harmonic mean (FLHHM) operator and developed an approach based on FLWHM and FLHHM operator to multiple attribute group decision making with triangular fuzzy linguistic variables.

All of the existing triangular fuzzy linguistic aggregation operators only consider situations where all the elements in the triangular fuzzy linguistic variables are independent. However, in many practical situations, the elements in the triangular fuzzy linguistic variables are usually correlative. Therefore, we need to find some new ways to deal with these situations in which the decision data in question are correlative. The Choquet integral (Choquet 1953) is a very useful way of measuring the expected utility of an uncertain event, and can be utilized to depict the correlations of the decision data under consideration.

Motivated by the correlation properties of the Choquet integral, in this paper we propose some triangular fuzzy linguistic aggregation operators, whose prominent characteristic is that they cannot only consider the importance of the elements or their ordered positions, but also reflect the correlations of the elements or their ordered positions. To do so, the remainder of this paper is set out as follows. In the next section, we introduce some basic concepts related to triangular fuzzy linguistic variables and Choquet integral and some operational laws of triangular fuzzy linguistic variables. In Section 2 we have developed three fuzzy linguistic Choquet integral aggregation operators: fuzzy linguistic choquet ordered averaging (FLCOA) operator, fuzzy linguistic choquet ordered geometric (FLCOG) operator and fuzzy linguistic choquet ordered harmonic mean (FLCOHM) operator and studied some desirable properties of these operators, such as commutativity, idempotency and monotonicity. In Section 3, we have developed an approach to multiple attribute decision making based on these operators with triangular fuzzy linguistic variables. In Section 4, an illustrative example is pointed out. In the last section we conclude the paper and give some remarks. 


\section{Preliminaries}

Let $S=\left\{s_{i} \mid i=1,2, \cdots, t\right\}$ be a linguistic term set with odd cardinality. Any label, $s_{i}$ represents a possible value for a linguistic variable, and it should satisfy the following characteristics: 1) The set is ordered: $s_{i}>s_{j}$, if $\left.i>j ; 2\right)$ There is the reciprocal operator: $\operatorname{rec}\left(s_{i}\right)=s_{j}$ such that $\left.i=t+1-j ; 3\right)$ Max operator: $\max \left(s_{i}, s_{j}\right)=s_{i}$, if $s_{i} \geq s_{j}$; 4) Min operator: $\min \left(s_{i}, s_{j}\right)=s_{i}$, if $s_{i} \leq s_{j}$. For example, $S$ can be defined as (Xu 2004a; Wei 2009a):

$S=\left\{s_{1}=\right.$ extremely poor, $s_{2}=$ very poor, $s_{3}=$ poor, $s_{4}=$ medium, $s_{5}=$ good,

$s_{6}=$ very good, $s_{7}=$ extremely good $\}$.

To preserve all the given information, we extend the discrete term set $\mathrm{S}$ to a continuous term set $\bar{S}=\left\{s_{a} \mid s_{1} \leq s_{a} \leq s_{q}, a \in[1, q]\right\}$, where $\mathrm{q}$ is a sufficiently large positive integer. If $s_{a} \in S$, then we call $s_{a}$ the original linguistic term, otherwise, we call $s_{a}$ the virtual linguistic term. In general, the decision maker uses the original linguistic term to evaluate attributes and alternatives, and the virtual linguistic terms can only appear in calculation (Xu 2004a; Wei 2009a).

Definition 1. Let $s_{\alpha}, s_{\beta} \in \bar{S}$, then we call:

$$
d\left(s_{\alpha}, s_{\beta}\right)=|\alpha-\beta|
$$

the distance between $s_{\alpha}$ and $s_{\beta}(\mathrm{Xu} 2007 \mathrm{a})$.

Since the decision maker is characterized by his own personal background and experience, in some situations, the decision maker may provide fuzzy linguistic information because of time pressure, lack of knowledge, and their limited expertise related with the problem domain. In the following we introduce the concept of triangular fuzzy linguistic variable.

Definition 2. Let $\tilde{s}=\left[s_{\alpha}, s_{\beta}, s_{\gamma}\right] \in \tilde{S}$, where $s_{\alpha}, s_{\beta}, s_{\gamma} \in \bar{S}, s_{\alpha}, s_{\beta}$ and $s_{\gamma}$ are the lower, modal and upper values of $\tilde{s}$, respectively, then we call $\tilde{s}$ a triangular fuzzy linguistic variable, which is characterized by the following member function (Xu 2007b):

$$
\mu_{\tilde{s}}(\theta)=\left\{\begin{array}{ll}
0, & s_{1} \leq s_{\theta} \leq s_{\alpha} \\
\frac{d\left(s_{\theta}, s_{\alpha}\right)}{d\left(s_{\beta}, s_{\alpha}\right)}, & s_{\alpha} \leq s_{\theta} \leq s_{\beta} \\
\frac{d\left(s_{\theta}, s_{\gamma}\right)}{d\left(s_{\beta}, s_{\gamma}\right)}, & s_{\beta} \leq s_{\theta} \leq s_{\gamma} \\
0, & s_{\gamma} \leq s_{\theta} \leq s_{q}
\end{array} .\right.
$$

Clearly, $s_{\beta}$ gives the maximal grade of $\mu_{\tilde{s}}(\theta)\left(\mu_{\tilde{s}}(\theta)=1\right), s_{\alpha}$ and $s_{\gamma}$ are the lower and upper bounds with limit the field of the possible evaluation. Especially, if $s_{\alpha}=s_{\beta}=s_{\gamma}$, then $\tilde{S}$ is reduced to a linguistic variable.

Let $\tilde{S}$ be the set of all triangular fuzzy linguistic variables. Consider any three triangular 
fuzzy linguistic variables $\tilde{s}=\left(s_{\alpha}, s_{\beta}, s_{\gamma}\right), \tilde{s}_{1}=\left(s_{\alpha_{1}}, s_{\beta_{1}}, s_{\gamma_{1}}\right), \tilde{s}_{2}=\left(s_{\alpha_{2}}, s_{\beta_{2}}, s_{\gamma_{2}}\right) \in \tilde{S}$, and suppose that $\lambda \in[0,1]$, then $\mathrm{Xu}(2007 \mathrm{~b})$ defined their operational laws as follows:

(1) $\tilde{s}_{1} \oplus \tilde{s}_{2}=\left(s_{\alpha_{1}}, s_{\beta_{1}}, s_{\gamma_{1}}\right) \oplus\left(s_{\alpha_{2}}, s_{\beta_{2}}, s_{\gamma_{2}}\right)=\left(s_{\alpha_{1}+\alpha_{2}}, s_{\beta_{1}+\beta_{2}}, s_{\gamma_{1}+\gamma_{2}}\right)$;

(2) $\tilde{s}_{1} \otimes \tilde{s}_{2}=\left(s_{\alpha_{1}}, s_{\beta_{1}}, s_{\gamma_{1}}\right) \otimes\left(s_{\alpha_{2}}, s_{\beta_{2}}, s_{\gamma_{2}}\right) \approx\left(s_{\alpha_{1} \alpha_{2}}, s_{\beta_{1} \beta_{2}}, s_{\gamma_{1} \gamma_{2}}\right)$;

(3) $1 / \tilde{s} \approx\left(s_{1 / \gamma}, s_{1 / \beta}, s_{1 / \alpha}\right)$;

(4) $\lambda \tilde{s}=\lambda \times\left(s_{\alpha}, s_{\beta}, s_{\gamma}\right)=\left(s_{\lambda \alpha}, s_{\lambda \beta}, s_{\lambda \gamma}\right)$;

(5) $\tilde{s}^{\lambda}=\left(s_{\alpha}, s_{\beta}, s_{\gamma}\right)^{\lambda}=\left(s_{\alpha^{\lambda}}, s_{\beta^{\lambda}}, s_{\gamma^{\lambda}}\right)$.

In the following, $\mathrm{Xu}$ (2007b) introduced a formula for comparing triangular fuzzy linguistic variables.

Definition 3. Let $\tilde{s}_{1}=\left(s_{\alpha_{1}}, s_{\beta_{1}}, s_{\gamma_{1}}\right), \tilde{s}_{2}=\left(s_{\alpha_{2}}, s_{\beta_{2}}, s_{\gamma_{2}}\right) \in \tilde{S}$, then the degree of possibility of $\tilde{s}_{1} \geq \tilde{s}_{2}$ is defined as [10]:

$$
\begin{aligned}
& p\left(\tilde{s}_{1} \geq \tilde{s}_{2}\right)= \\
& \lambda \max \left\{1-\max \left[\frac{d\left(s_{\beta_{2}}, s_{\alpha_{1}}\right)}{d\left(s_{\beta_{1}}, s_{\alpha_{1}}\right)+d\left(s_{\beta_{2}}, s_{\alpha_{2}}\right)}, 0\right], 0\right\}+ \\
& (1-\lambda) \max \left\{1-\max \left[\frac{d\left(s_{\gamma_{2}}, s_{\beta_{1}}\right)}{d\left(s_{\gamma_{1}}, s_{\beta_{1}}\right)+d\left(s_{\gamma_{2}}, s_{\beta_{2}}\right)}, 0\right], 0\right\},
\end{aligned}
$$

where the value $\lambda$ is an index of rating attitude. It reflects the decision maker's riskbearing attitude. If $\lambda<0.5$, the decision maker is risk lover. If $\lambda=0.5$, the decision maker is neutral to risk. If $\lambda>0.5$, the decision maker is risk avertor. Especially, if both the linguistic variables $\tilde{s}_{1}$ and $\tilde{s}_{2}$ express precise linguistic information, i.e. if:

$$
d\left(s_{\gamma_{1}}, s_{\alpha_{1}}\right)+d\left(s_{\gamma_{2}}, s_{\alpha_{2}}\right)=0
$$

then we define the degree of possibility of $\tilde{s}_{1} \geq \tilde{s}_{2}$ as:

$$
p\left(\tilde{s}_{1} \geq \tilde{s}_{2}\right)=\left\{\begin{array}{ccc}
1 & \text { if } & \tilde{s}_{1}>\tilde{s}_{2} \\
0.5 & \text { if } & \tilde{s}_{1}=\tilde{s}_{2} . \\
0 & \text { if } & \tilde{s}_{1}<\tilde{s}_{2}
\end{array}\right.
$$

From Definition 3, we can easily get the following results easily:

(1) $0 \leq p\left(\tilde{s}_{1} \geq \tilde{s}_{2}\right) \leq 1,0 \leq p\left(\tilde{s}_{2} \geq \tilde{s}_{1}\right) \leq 1$;

(2) $p\left(\tilde{s}_{1} \geq \tilde{s}_{2}\right)+p\left(\tilde{s}_{2} \geq \tilde{s}_{1}\right)=1$. Especially, $p\left(\tilde{s}_{1} \geq \tilde{s}_{1}\right)=p\left(\tilde{s}_{2} \geq \tilde{s}_{2}\right)=0.5$.

However, the above aggregation operators with triangular fuzzy linguistic variable is based on the assumption that the attribute of decision makers are independent, which is characterized by an independence axiom (Keeney, Raiffa 1976), that is, these operators are based on the implicit assumption that attributes of decision makers are independent 
of one another; their effects are viewed as additive. For real decision making problems, there is always some degree of inter-dependent characteristics between attributes. Usually, there is interaction among attributes of decision makers. However, this assumption is too strong to match decision behaviors in the real world. The independence axiom generally cannot be satisfied. Thus, it is necessary to consider this issue.

Let $\mu\left(x_{i}\right)(i=1,2, \cdots, n)$ be the weight of the elements $x_{i} \in X(i=1,2, \cdots, n)$, where $\mu$ is a fuzzy measure, defined as follows:

Definition 4 (Wang et al. 1992). A fuzzy measure $\mu$ on the set $X$ is a set function $\mu: \theta(x) \rightarrow[0,1]$ satisfying the following axioms:

(1) $\mu(\phi)=0, \mu(X)=1$;

(2) $A \subseteq B$ implies $\mu(A) \leq \mu(B)$, for all $A, B \subseteq X$;

(3) $\mu(A \cup B)=\mu(A)+\mu(B)+\rho \mu(A) \mu(B)$, for all $A, B \subseteq X$ and $A \cap B=\phi$, where $\rho \in(-1, \infty)$.

Especially, if $\rho=0$, then the condition (3) reduces to the axiom of additive measure:

$$
\mu(A \cup B)=\mu(A)+\mu(B) \text {, for all } A, B \subseteq X \text { and } A \cap B=\phi .
$$

If all the elements in $X$ are independent, and we have:

$$
\mu(A)=\sum_{x_{i} \in A} \mu\left(\left\{x_{i}\right\}\right), \text { for all } A \subseteq X .
$$

Definition 5 (Grabisch et al. 2000). Let $f$ be a positive real-valued function on $X$, and $\mu$ be a fuzzy measure on $X$. The discrete Choquet integral of $f$ with respective to $\mu$ is defined by:

$$
C_{\mu}(f)=\sum_{i=1}^{n} f_{\sigma(i)}\left[\mu\left(A_{\sigma(i)}\right)-\mu\left(A_{\sigma(i-1)}\right)\right],
$$

where $(\sigma(1), \sigma(2), \cdots, \sigma(n))$ is a permutation of $(1,2, \cdots, n)$, such that $f_{\sigma(i-1)} \geq f_{\sigma(i)}$ for all $j=2, \cdots, n, A_{\sigma(k)}=\left\{x_{\sigma(j)} \mid j \leq k\right\}$, for $k \geq 1$, and $A_{\sigma(0)}=\phi$.

It is seen that the discrete Choquet integral is a linear expression up to a reordering of the elements.

\section{Some choquet ordered aggregating operators with triangular fuzzy linguistic information}

$\mathrm{Xu}$ (2004) extend the OWA operator (Yager 1988, 2004) to triangular fuzzy linguistic setting and proposed the fuzzy linguistic ordered weighted averaging (FLOWA) operator.

Definition 6. Let $\tilde{s}_{i}(i=1,2, \cdots, n)$ be a set of triangular fuzzy linguistic variables, and:

$$
\operatorname{FLOWA}_{w}\left(\tilde{s}_{1}, \tilde{s}_{2}, \cdots, \tilde{s}_{n}\right)=\bigoplus_{j=1}^{n}\left(w_{j} \otimes \tilde{s}_{\sigma(j)}\right),
$$

where $w=\left(w_{1}, w_{2}, \cdots, w_{n}\right)$ is the associated exponential weighting vector, with 
$w_{j} \in[0,1], \sum_{j=1}^{n} w_{j}=1$, and $(\sigma(1), \sigma(2), \cdots, \sigma(n))$ is a permutation of $(1,2, \cdots, n)$, such that $\tilde{s}_{\sigma(j-1)} \geq \tilde{s}_{\sigma(j)}$ for all $j=2, \cdots, n$, then the function FLOWA is called the fuzzy linguistic variables ordered weighted averaging (FLOWA) operator of dimension $n$.

To rank these triangular fuzzy linguistic variables $\tilde{s}_{j}(j=1,2, \cdots, n)$, we first compare each argument $\tilde{s}_{i}$ with all the triangular fuzzy linguistic variables $\tilde{s}_{j}(j=1,2, \cdots, n)$ by using Eq. (3). For simplicity, we let $p_{i j}=p\left(\tilde{s}_{i} \geq \tilde{s}_{j}\right)$, then we develop a complementary matrix as $P=\left(p_{i j}\right)_{n \times n}$, where:

$$
p_{i j} \geq 0, p_{i j}+p_{j i}=1, p_{i i}=0.5, i, j=1,2, \cdots, n .
$$

Summing all the elements in each line of matrix $P$, we get:

$$
p_{i}=\sum_{j=1}^{n} p_{i j}, i=1,2, \cdots, n \text {. }
$$

Then we rank the arguments $\tilde{s}_{j}$ in descending order in accordance with the values of $p_{i}(i=1,2, \cdots, n)$.

Zhao and Wei (2009) developed the fuzzy linguistic variables ordered weighted geometric (FLOWG) operator.

Definition 7. Let $\tilde{s}_{i}(i=1,2, \cdots, n)$ be a set of triangular fuzzy linguistic variables, and:

$$
\operatorname{FLOWG}_{w}\left(\tilde{s}_{1}, \tilde{s}_{2}, \cdots, \tilde{s}_{n}\right)=\bigotimes_{j=1}^{n}\left(\tilde{s}_{\sigma(j)}\right)^{w_{j}},
$$

where $w=\left(w_{1}, w_{2}, \cdots, w_{n}\right)$ is the associated exponential weighting vector, with $w_{j} \in[0,1], \sum_{j=1}^{n} w_{j}=1$, and $(\sigma(1), \sigma(2), \cdots, \sigma(n))$ is a permutation of $(1,2, \cdots, n)$, such that $\tilde{s}_{\sigma(j-1)} \geq \tilde{s}_{\sigma(j)}$ for all $j=2, \cdots, n$, then the function FLOWG is called the fuzzy linguistic variables ordered weighted geometric (FLOWG) operator of dimension $n$.

Motivated by the basic idea of the harmonic mean operators (Xu 2008, 2009a), Wei (2009c) developed the fuzzy linguistic ordered weighted harmonic mean (FLOWHM) operator.

Definition 8. Let $\tilde{s}_{i}(i=1,2, \cdots, n)$ be a set of triangular fuzzy linguistic variables, and:

$$
\operatorname{FLOWHM}_{w}\left(\tilde{s}_{1}, \tilde{s}_{2}, \cdots, \tilde{s}_{n}\right)=\frac{1}{\bigoplus_{j=1}^{n}\left(\frac{w_{j}}{\tilde{s}_{\sigma(j)}}\right)},
$$

where $w=\left(w_{1}, w_{2}, \cdots, w_{n}\right)$ is the associated weighting vector, with $w_{j} \in[0,1], \sum_{j=1}^{n} w_{j}=1$, and $(\sigma(1), \sigma(2), \cdots, \sigma(n))$ is a permutation of $(1,2, \cdots, n)$, such that $\tilde{s}_{\sigma(j-1)} \geq \tilde{s}_{\sigma(j)}$ for all $j=2, \cdots, n$, then the function FLOWHM is called the fuzzy linguistic ordered weighted harmonic mean (FLOWHM) operator of dimension $n$.

Based on Definition 5 and 6, in what follows, we shall develop the fuzzy linguistic choquet ordered averaging (FLCOA) operator based on the well-known Choquet integral (Choquet 1953). 
Definition 9. Let $\tilde{s}_{i}(i=1,2, \cdots, n)$ be a set of triangular fuzzy linguistic variables on $X$, and $\mu$ be a fuzzy measure on $X$, then we call:

$$
\operatorname{FLCOA}_{\mu}\left(\tilde{s}_{1}, \tilde{s}_{2}, \cdots, \tilde{s}_{n}\right)=\bigoplus_{j=1}^{n}\left(\left(\mu\left(A_{\sigma(j)}\right)-\mu\left(A_{\sigma(j-1)}\right)\right) \otimes \tilde{s}_{\sigma(j)}\right)
$$

the fuzzy linguistic choquet ordered averaging (FLCOA) operator, where $(\sigma(1), \sigma(2), \cdots, \sigma(n))$ is a permutation of $(1,2, \cdots, n)$, such that $\tilde{s}_{\sigma(j-1)} \geq \tilde{s}_{\sigma(j)}$ for all $j=2, \cdots, n, A_{\sigma(k)}=\left\{x_{\sigma(j)} \mid j \leq k\right\}$, for $k \geq 1$, and $A_{\sigma(0)}=\phi$.

Especially, if $\mu\left(\left\{x_{\sigma(j)}\right\}\right)=\mu\left(A_{\sigma(j)}\right)-\mu\left(A_{\sigma(j-1)}\right), j=1,2, \cdots, n$, then FLCOA operator reduce to FLWA operator. If $\mu(A)=\sum_{x_{j} \in A} \mu\left(\left\{x_{j}\right\}\right)$, for all $A \subseteq X$, where $|A|$ is the number of the elements in the set $A$, then $w_{j}=\mu\left(A_{\sigma(j)}\right)-\mu\left(A_{\sigma(j-1)}\right), i=1,2, \cdots, n$, where $w=\left(w_{1}, w_{2}, \cdots, w_{n}\right)^{T}, w_{j} \geq 0, j=1,2, \cdots, n$, and $\sum_{j=1}^{n} w_{j}=1$, then, FLCOA operator re-
duce to FLOWA operator.

It's easy to prove that the FLCOA operator has the following properties.

Theorem 1 (Commutativity).

$$
\operatorname{FLCOA}_{\mu}\left(\tilde{s}_{1}, \tilde{s}_{2}, \cdots, \tilde{s}_{n}\right)=\operatorname{FLCOA}_{\mu}\left(\tilde{s}_{1}^{\prime}, \tilde{s}_{2}^{\prime}, \cdots, \tilde{s}_{n}^{\prime}\right),
$$

where $\left(\tilde{s}_{1}^{\prime}, \tilde{s}_{2}^{\prime}, \cdots, \tilde{s}_{n}^{\prime}\right)$ is any permutation of $\left(\tilde{s}_{1}, \tilde{s}_{2}, \cdots, \tilde{s}_{n}\right)$.

Theorem 2 (Idempotency). If $\tilde{s}_{j}=\tilde{s}$ for all $j$, then:

$$
\operatorname{FLCOA}_{\mu}\left(\tilde{s}_{1}, \tilde{s}_{2}, \cdots, \tilde{s}_{n}\right)=\tilde{s} .
$$

Theorem 3 (Monotonicity). If $\tilde{s}_{j} \leq \tilde{s}_{j}^{\prime}$ for all $j$, then:

$$
\operatorname{FLCOA}_{\mu}\left(\tilde{s}_{1}, \tilde{s}_{2}, \cdots, \tilde{s}_{n}\right) \leq \operatorname{FLCOA}_{\mu}\left(\tilde{s}_{1}^{\prime}, \tilde{s}_{2}^{\prime}, \cdots, \tilde{s}_{n}^{\prime}\right) .
$$

Based on Definition 5 and 7-8, in what follows, we shall develop the fuzzy linguistic choquet ordered geometric (FLCOG) operator and fuzzy linguistic choquet ordered harmonic (FLCOG) operator based on the well-known Choquet integral (Choquet 1953).

Definition 10. Let $\tilde{s}_{i}(i=1,2, \cdots, n)$ be a set of triangular fuzzy linguistic variables on $X$, and $\mu$ be a fuzzy measure on $X$, then we call:

$$
\operatorname{FLOWG}_{\mu}\left(\tilde{s}_{1}, \tilde{s}_{2}, \cdots, \tilde{s}_{n}\right)=\bigotimes_{j=1}^{n}\left(\tilde{s}_{\sigma(j)}\right)^{\mu\left(A_{\sigma(j)}\right)-\mu\left(A_{\sigma(j-1)}\right)}
$$

the fuzzy linguistic choquet ordered geometric (FLCOG) operator, where $(\sigma(1), \sigma(2), \cdots, \sigma(n))$ is a permutation of $(1,2, \cdots, n)$, such that $\tilde{s}_{\sigma(j-1)} \geq \tilde{s}_{\sigma(j)}$ for all $j=2, \cdots, n, A_{\sigma(k)}=\left\{x_{\sigma(j)} \mid j \leq k\right\}$, for $k \geq 1$, and $A_{\sigma(0)}=\phi$.

Especially, if $\mu\left(\left\{x_{\sigma(j)}\right\}\right)=\mu\left(A_{\sigma(j)}\right)-\mu\left(A_{\sigma(j-1)}\right), j=1,2, \cdots, n$, then FLCOG operator reduce to FLWG operator. If $\mu(A)=\sum_{x_{j} \in A} \mu\left(\left\{x_{j}\right\}\right)$, for all $A \subseteq X$, where $|A|$ is the num- 
ber of the elements in the set $A$, then $w_{j}=\mu\left(A_{\sigma(j)}\right)-\mu\left(A_{\sigma(j-1)}\right), i=1,2, \cdots, n$, where $w=\left(w_{1}, w_{2}, \cdots, w_{n}\right)^{T}, w_{j} \geq 0, j=1,2, \cdots, n$, and $\sum_{j=1}^{n} w_{j}=1$, then, FLCOG operator re-
duce to FLOWG operator.

Definition 11. Let $\tilde{s}_{i}(i=1,2, \cdots, n)$ be a set of triangular fuzzy linguistic variables on $X$, and $\mu$ be a fuzzy measure on $X$, then we call:

$$
\operatorname{FLOWHM}_{\mu}\left(\tilde{s}_{1}, \tilde{s}_{2}, \cdots, \tilde{s}_{n}\right)=\frac{1}{\bigoplus_{j=1}^{n}\left(\frac{\mu\left(A_{\sigma(j)}\right)-\mu\left(A_{\sigma(j-1)}\right)}{\tilde{s}_{\sigma(j)}}\right)}
$$

the fuzzy linguistic choquet ordered harmonic mean (FLCOHM) operator, where $(\sigma(1), \sigma(2), \cdots, \sigma(n))$ is a permutation of $(1,2, \cdots, n)$, such that $\tilde{s}_{\sigma(j-1)} \geq \tilde{s}_{\sigma(j)}$ for all $j=2, \cdots, n, A_{\sigma(k)}=\left\{x_{\sigma(j)} \mid j \leq k\right\}$, for $k \geq 1$, and $A_{\sigma(0)}=\phi$.

Especially, if $\mu\left(\left\{x_{\sigma(j)}\right\}\right)=\mu\left(A_{\sigma(j)}\right)-\mu\left(A_{\sigma(j-1)}\right), j=1,2, \cdots, n$, then FLCOHM operator reduces to FLWHA operator. If $\mu(A)=\sum_{x_{j} \in A} \mu\left(\left\{x_{j}\right\}\right)$, for all $A \subseteq X$, where $|A|$ is the number of the elements in the set $A$, then $w_{j}=\mu\left(A_{\sigma(j)}\right)-\mu\left(A_{\sigma(j-1)}\right), i=1,2, \cdots, n$, where $w=\left(w_{1}, w_{2}, \cdots, w_{n}\right)^{T}, w_{j} \geq 0, j=1,2, \cdots, n$, and $\sum_{j=1}^{n} w_{j}=1$, then FLCOHM op-
erator reduces to FLOWHM operator.

It's easy to prove that the FLCOG operator and FLCOHM have the properties: commutativity, idempotency and monotonicity.

\section{An approach to multiple attribute decision making with triangular fuzzy linguistic variable}

In this section, we shall develop some approaches to multiple attribute decision making with triangular fuzzy linguistic variables as follows.

Let $A=\left\{A_{1}, A_{2}, \cdots, A_{m}\right\}$ be a discrete set of alternatives, and $G=\left\{G_{1}, G_{2}, \cdots, G_{n}\right\}$ be the set of attributes, $\omega=\left(\omega_{1}, \omega_{2}, \cdots, \omega_{n}\right)$ is the weighting vector of the attribute $G_{j}(j=1,2, \cdots, n)$, where $\omega_{j} \in[0,1], \sum_{j=1}^{n} \omega_{j}=1$. Suppose that $\tilde{R}=\left(\tilde{r}_{i j}\right)_{m \times n}$ is the fuzzy linguistic decision matrix, where $\tilde{r}_{i j} \in \tilde{S}$ is a preference value, which take the form of triangular fuzzy linguistic variable, given by the decision maker, for the alternative $A_{i} \in A$ with respect to the attribute $G_{j} \in G, i=1,2, \cdots, m, j=1,2, \cdots, n$.

In the following, we apply the FNIFCOA and FNIFCOGM operator to multiple attribute decision making with triangular fuzzy linguistic information.

Step 1. Utilize the decision information given in matrix $\tilde{R}$, and the FLCOA operator:

$$
\tilde{r}_{i}=\operatorname{FLCOA}\left(\tilde{r}_{i 1}, \tilde{r}_{i 2}, \cdots, \tilde{r}_{i n}\right), i=1,2, \cdots, m
$$

to derive the overall preference values $\tilde{r}_{i}(i=1,2, \cdots, m)$ of the alternative $A_{i}$. 
Or utilize the decision information given in matrix $\tilde{R}$, and the FLCOG operator:

$$
\tilde{r}_{i}=\operatorname{FLCOG}\left(\tilde{r}_{i 1}, \tilde{r}_{i 2}, \cdots, \tilde{r}_{i n}\right), i=1,2, \cdots, m
$$

to derive the overall preference values $\tilde{r}_{i}(i=1,2, \cdots, m)$ of the alternative $A_{i}$.

Or utilize the decision information given in matrix $\tilde{R}$, and the FLCOHM operator:

$$
\tilde{r}_{i}=\operatorname{FLCOHM}\left(\tilde{r}_{i 1}, \tilde{r}_{i 2}, \cdots, \tilde{r}_{i n}\right), i=1,2, \cdots, m
$$

to derive the overall preference values $\tilde{r}_{i}(i=1,2, \cdots, m)$ of the alternative $A_{i}$.

Step 2. To rank these collective overall preference values $\tilde{r}_{i}(i=1,2, \cdots, m)$, we first compare each $\tilde{r}_{i}$ with all the $\tilde{r}_{j}(j=1,2, \cdots, m)$ by using Eq. (3). For simplicity, we let $p_{i j}=p\left(\tilde{r}_{i} \geq \tilde{r}_{j}\right)$, then we develop a complementary matrix as $P=\left(p_{i j}\right)_{m \times m}$, where $p_{i j} \geq 0, p_{i j}+p_{j i}=1, p_{i i}=0.5, i, j=1,2, \cdots, m$.

Summing all the elements in each line of matrix $P$, we have:

$$
p_{i}=\sum_{j=1}^{m} p_{i j}, i=1,2, \cdots, m .
$$

Then we rank the collective overall preference values $\tilde{r}_{i}(i=1,2, \cdots, m)$ in descending order in accordance with the values of $p_{i}(i=1,2, \cdots, m)$.

Step 3. Rank all the alternatives $A_{i}(i=1,2, \cdots, m)$ and select the best one(s) in accordance with the collective overall preference values $\tilde{r}_{i}(i=1,2, \cdots, m)$.

Step 4. End.

\section{Illustrative example}

Thus, in this section we shall present a numerical example to show the performance evaluation of emergency management with triangular fuzzy linguistic information in order to illustrate the method proposed in this paper. There is a panel with four possible emerging technology enterprises $A_{i}(i=1,2,3,4,5)$ to select. The experts selects four attribute to evaluate the performance evaluation of emergency management of five possible emerging technology enterprises: 1) $G_{1}$ is the decision command system performance; 2) $G_{2}$ is the emergency response system performance; 3) $G_{3}$ is the emergency preset system performance; 4) $\mathrm{G}_{4}$ is the resources guarantee system performance. The performance evaluation of emergency management of five possible emerging technology enterprises $A_{i}(i=1,2, \cdots, 5)$ are to be evaluated using the triangular fuzzy linguistic variable by the decision maker under the above four attributes, and construct the decision matrix as listed in the following matrix $\tilde{R}=\left(\tilde{r}_{i j}\right)_{5 \times 4}$ is presented in Table 1 .

Then, we utilize the approach developed to get the most desirable emerging technology enterprise(s) with the best performance evaluation of emergency management.

Step 1. Suppose the fuzzy measure of attribute of $G_{j}(j=1,2, \cdots, n)$ and attribute sets of $G$ as follows:

$$
\begin{aligned}
& \mu\left(G_{1}\right)=0.30, \mu\left(G_{2}\right)=0.35, \mu\left(G_{3}\right)=0.30, \mu\left(G_{4}\right)=0.22, \mu\left(G_{1}, G_{2}\right)=0.70, \\
& \mu\left(G_{1}, G_{3}\right)=0.60, \mu\left(G_{1}, G_{4}\right)=0.55, \mu\left(G_{2}, G_{3}\right)=0.50, \mu\left(G_{2}, G_{4}\right)=0.45,
\end{aligned}
$$




$$
\begin{aligned}
& \mu\left(G_{3}, G_{4}\right)=0.40, \mu\left(G_{1}, G_{2}, G_{3}\right)=0.82, \mu\left(G_{1}, G_{2}, G_{4}\right)=0.87, \\
& \mu\left(G_{1}, G_{3}, G_{4}\right)=0.75, \mu\left(G_{2}, G_{3}, G_{4}\right)=0.60, \mu\left(G_{1}, G_{2}, G_{3}, G_{4}\right)=1.00 .
\end{aligned}
$$

Step 2. By utilizing the decision information given in Table 1, and the FLCOA, FLCOG and FLCOHM operators, we derive the overall values of performance evaluation of emergency management of the emerging technology enterprises. The aggregating results are shown in Table 2.

Step 3. According to the aggregating results shown in Table 2 and the formula of degree of possibility (3), the ordering of the alternatives are shown in Table 10. Note that > means "preferred to". As we can see from Table 3, depending on the aggregation operators used, the ordering of the five emerging technology enterprises is the same, and the best emerging technology enterprises with the best performance evaluation of emergency managementis $\mathrm{A}_{5}$.

Table 1. Triangular fuzzy linguistic decision matrix

\begin{tabular}{ccccc}
\hline & $\mathrm{G}_{1}$ & $\mathrm{G}_{2}$ & $\mathrm{G}_{3}$ & $\mathrm{G}_{4}$ \\
\hline $\mathrm{A}_{1}$ & $\left(s_{2}, s_{3}, s_{4}\right)$ & $\left(s_{3}, s_{4}, s_{5}\right)$ & $\left(s_{1}, s_{2}, s_{4}\right)$ & $\left(s_{1}, s_{2}, s_{3}\right)$ \\
\hline $\mathrm{A}_{2}$ & $\left(s_{1}, s_{2}, s_{3}\right)$ & $\left(s_{4}, s_{5}, s_{7}\right)$ & $\left(s_{3}, s_{4}, s_{5}\right)$ & $\left(s_{2}, s_{3}, s_{4}\right)$ \\
\hline $\mathrm{A}_{3}$ & $\left(s_{2}, s_{4}, s_{5}\right)$ & $\left(s_{1}, s_{3}, s_{4}\right)$ & $\left(s_{4}, s_{5}, s_{6}\right)$ & $\left(s_{3}, s_{4}, s_{7}\right)$ \\
\hline $\mathrm{A}_{4}$ & $\left(s_{4}, s_{5}, s_{6}\right)$ & $\left(s_{1}, s_{2}, s_{3}\right)$ & $\left(s_{2}, s_{3}, s_{4}\right)$ & $\left(s_{2}, s_{3}, s_{5}\right)$ \\
\hline $\mathrm{A}_{5}$ & $\left(s_{3}, s_{4}, s_{5}\right)$ & $\left(s_{4}, s_{6}, s_{7}\right)$ & $\left(s_{1}, s_{4}, s_{5}\right)$ & $\left(s_{3}, s_{4}, s_{6}\right)$ \\
\hline
\end{tabular}

Table 2. The overall performance evaluation values of the emerging technology enterprises

\begin{tabular}{cccc}
\hline & FLCOA & FLCOG & FLCOHM \\
\hline $\mathrm{A}_{1}$ & $\left(s_{2.05}, s_{3.05}, s_{4.17}\right)$ & $\left(s_{1.87}, s_{2.94}, s_{4.11}\right)$ & $\left(s_{1.69}, s_{2.82}, s_{4.04}\right)$ \\
\hline $\mathrm{A}_{2}$ & $\left(s_{2.45}, s_{3.45}, s_{4.80}\right)$ & $\left(s_{2.05}, s_{3.18}, s_{4.48}\right)$ & $\left(s_{1.70}, s_{2.93}, s_{4.20}\right)$ \\
\hline $\mathrm{A}_{3}$ & $\left(s_{2.45}, s_{4.05}, s_{5.25}\right)$ & $\left(s_{2.16}, s_{3.98}, s_{5.17}\right)$ & $\left(s_{1.88}, s_{3.91}, s_{5.08}\right)$ \\
\hline $\mathrm{A}_{4}$ & $\left(s_{2.75}, s_{3.75}, s_{5.00}\right)$ & $\left(s_{2.38}, s_{3.50}, s_{4.82}\right)$ & $\left(s_{2.00}, s_{3.24}, s_{4.62}\right)$ \\
\hline $\mathrm{A}_{5}$ & $\left(s_{2.79}, s_{4.30}, s_{5.30}\right)$ & $\left(s_{2.58}, s_{4.01}, s_{4.88}\right)$ & $\left(s_{2.80}, s_{5.11}, s_{6.38}\right)$ \\
\hline
\end{tabular}

Table 3. Ordering of the alternative by utilizing the FLCOA, FLCOG and FLCOHM operators

\begin{tabular}{cc}
\hline & Ordering \\
\hline FLCOA & $\mathrm{A}_{5}>\mathrm{A}_{3}>\mathrm{A}_{4}>\mathrm{A}_{2}>\mathrm{A}_{1}$ \\
\hline FLCOG & $\mathrm{A}_{5}>\mathrm{A}_{3}>\mathrm{A}_{4}>\mathrm{A}_{2}>\mathrm{A}_{1}$ \\
\hline FLCOHM & $\mathrm{A}_{5}>\mathrm{A}_{3}>\mathrm{A}_{4}>\mathrm{A}_{2}>\mathrm{A}_{1}$ \\
\hline
\end{tabular}


Especially, if the triangular fuzzy linguistic variable $\tilde{s}=\left[s_{\alpha}, s_{\beta}, s_{\gamma}\right]$ are reduced to the uncertain linguistic variable $\tilde{s}=\left[s_{\alpha}, s_{\gamma}\right]$, then the FLCOA, FLCOG and FLCOHM operators are reduced to the uncertain linguistic choquet ordered averaging (ULCOA) operator (Xu 2009), uncertain linguistic choquet ordered geometric (ULCOG) operator (Xu 2009) and uncertain linguistic choquet ordered harmonic mean (ULCOHM) operator; if $s_{\alpha}=s_{\beta}=s_{\gamma}$ then the FLCOA, FLCOG and FLCOHM operators are reduced to the linguistic choquet ordered averaging (LCOA) operator (Xu 2009), linguistic choquet ordered geometric (LCOG) operator (Xu 2009) and linguistic choquet ordered harmonic (LCOHM) operator.

\section{Conclusions}

The traditional Choquet integral aggregation operators are generally suitable for aggregating the information taking the form of numerical values, and yet they will fail in dealing with triangular fuzzy linguistic variable. In this paper, we have developed three fuzzy linguistic Choquet integral aggregation operators: fuzzy linguistic choquet ordered averaging (FLCOA) operator, fuzzy linguistic choquet ordered geometric (FLCOG) operator and fuzzy linguistic choquet ordered harmonic mean (FLCOHM) operator. The prominent characteristic of the operators is that they cannot only consider the importance of the elements or their ordered positions, but also reflect the correlation among the elements or their ordered positions. We have studied some desirable properties of these operators, such as commutativity, idempotency and monotonicity, and applied these operators to multiple attribute decision making with triangular fuzzy linguistic information. Finally, an illustrative example has been given to show the developed method. In the future, we shall continue working in the application of the triangular fuzzy linguistic multiple attribute decision making to other domains.

\section{Acknowledgement}

The work was supported by the Social Sciences Foundation of Ministry of Education of the People's Republic of China under Grant No. 14YJCZH091.

\section{References}

Auephanwiriyakul, S.; Keller, J. M. 2001. A comparison of the linguistic Choquet and Sugeno fuzzy integrals, in Proceedings of the Tenth IEEE International Conference on Fuzzy Systems, December 2001, Melbourne, Australia.

Choquet, G. 1953. Theory of capacities, Annal Institute Fourier 5: 131-295.

http://dx.doi.org/10.5802/aif.53

Grabisch, M.; Murofushi, T.; Sugeno, M. 2000. Fuzzy measure and integrals. New York: PhysicaVerlag.

Keeney, R. L.; Raiffa, H. 1976. Decision with multiple objectives. New York: Wiley.

Liu, P. D. 2009a. Multi-attribute decision-making method research based on interval vague set and TOPSIS method, Technological and Economic Development of Economy 15(3): 453-463. http://dx.doi.org/10.3846/1392-8619.2009.15.453-463 
Liu, P. D. 2009b. A novel method for hybrid multiple attribute decision making, KnowledgeBased Systems 22(5): 388-391. http://dx.doi.org/10.1016/j.knosys.2009.02.001

Liu, P. D.; Su Y. 2010. The multiple-attribute decision making method based on the FLHOWA operator, Computers \& Mathematics with Applications 60(9): 2609-2615.

http://dx.doi.org/10.1016/j.camwa.2010.08.087

Zhang, X.; Liu, P. 2010a. Method for aggregating triangular fuzzy intuitionistic fuzzy information and its application to decision making, Technological and Economic Development of Economy 16(2) 280-290. http://dx.doi.org/10.3846/tede.2010.18

Zhang, X.; Liu, P. 2010b. Method for multiple attribute decision-making under risk with interval numbers, International Journal of Fuzzy Systems 12(3): 237-242.

Liu, P.; Zhang, X. 2010. The study on multi-attribute decision-making with risk based on linguistic variable, International Journal of Computational Intelligence Systems 3(5): 601-609.

http://dx.doi.org/10.1080/18756891.2010.9727726

Liu, P.; Wang, Y. 2011. A multiple attribute group decision making method based on generalized interval-valued trapezoidal fuzzy numbers, Control and Cybernetics 40(1): 163-184.

Liu, P.; Zhang, X. 2011. Research on the supplier selection of supply chain based on entropy weight and improved ELECTRE-III method, International Journal of Production Research 49(3): 637-646. http://dx.doi.org/10.1080/00207540903490171

Liu, P.; Zhang, X.; Liu, W. 2011a. A risk evaluation method for the high-tech project investment based on uncertain linguistic variables, Technological Forecasting and Social Change 78(1): 40-50. http://dx.doi.org/10.1016/j.techfore.2010.04.011

Liu, P.; Jin, F.; Zhang, X.; Su, Y.; Wang, M. 2011b. Research on the multi-attribute decision-making under risk with interval probability based on prospect theory and the uncertain linguistic variables, Knowledge-Based Systems 24(4): 554-561. http://dx.doi.org/10.1016/j.knosys.2011.01.010 Merigó, J. M. 2010. Fuzzy decision making with immediate probabilities, Computers \& Industrial Engineering 58(4): 651-657. http://dx.doi.org/10.1016/j.cie.2010.01.007

Merigó, J. M.; Casanovas, M. 2009. Induced aggregation operators in decision making with the Dempster-Shafer belief structure, International Journal of Intelligent Systems 24(8): 934-954. http://dx.doi.org/10.1002/int.20368

Merigó, J. M.; Casanovas, M. 2010. Decision making with distance measures and linguistic aggregation operators, International Journal of Fuzzy Systems 12(3): 190-198.

Merigó, J. M.; Gil-Lafuente, A. M. 2009. The induced generalized OWA operator, Information Sciences 179: 729-741. http://dx.doi.org/10.1016/j.ins.2008.11.013

Merigó, J. M.; Gil-Lafuente, A. M. 2010. New decision-making techniques and their application in the selection of financial products, Information Sciences 180(11): 2085-2094.

http://dx.doi.org/10.1016/j.ins.2010.01.028

Merigó, J. M.; Casanovas, M.; Martínez, L. 2010. Linguistic aggregation operators for linguistic decision making based on the Dempster-Shafer theory of evidence, International Journal of Uncertainty, Fuzziness and Knowledge-Based Systems 18(3): 287-304. http://dx.doi.org/10.1142/ S0218488510006544

Tan, C.Q.; Chen, X. H. 2010. Intuitionistic fuzzy Choquet integral operator for multi-criteria decision making, Expert Systems with Applications 37(1): 149-157.

http://dx.doi.org/10.1016/j.eswa.2009.05.005

Wang, W. P. 2009a. Evaluating new product development performance by fuzzy linguistic computing, Expert Systems with Applications 36(6): 9759-9766.

http://dx.doi.org/10.1016/j.eswa.2009.02.034 
Wang, W. P. 2009b. Toward developing agility evaluation of mass customization systems using 2-tuple linguistic computing, Expert Systems with Applications 36: 3439-3447.

http://dx.doi.org/10.1016/j.eswa.2008.02.015

Wang, Z.; Klir, G. 1992. Fuzzy measure theory. New York: Plenum Press.

http://dx.doi.org/10.1007/978-1-4757-5303-5

Wei, G. W. 2008. Maximizing deviation method for multiple attribute decision making in intuitionistic fuzzy setting, Knowledge-Based Systems 21(8): 833-836.

http://dx.doi.org/10.1016/j.knosys.2008.03.038

Wei, G. W. 2009a. Uncertain linguistic hybrid geometric mean operator and its application to group decision making under uncertain linguistic environment, International Journal of Uncertainty, Fuzziness, Knowledge-Based Systems 17(2): 251-267.

http://dx.doi.org/10.1142/S021848850900584X

Wei, G. W. 2009b. Some geometric aggregation functions and their application to dynamic multiple attribute decision making in intuitionistic fuzzy setting, International Journal of Uncertainty, Fuzziness and Knowledge-Based Systems 17(2): 179-196.

http://dx.doi.org/10.1142/S0218488509005802

Wei, G. W. 2009c. Fuzzy linguistic hybrid harmonic mean operator and its application to software selection, Journal of Software 4(9): 1037-1042.

Wei, G. W. 2010a. A method for multiple attribute group decision making based on the ET-WG and ET-OWG operators with 2-tuple linguistic information, Expert Systems with Applications 37(12): 7895-7900. http://dx.doi.org/10.1016/j.eswa.2010.04.047

Wei, G. W. 2010b. Extension of TOPSIS method for 2-tuple linguistic multiple attribute group decision making with incomplete weight information, Knowledge and Information Systems 25: 623-634. http://dx.doi.org/10.1007/s10115-009-0258-3

Wei, G. W. 2010c. Some induced geometric aggregation operators with intuitionistic fuzzy information and their application to group decision making, Applied Soft Computing 10(2): 423-431. http://dx.doi.org/10.1016/j.asoc.2009.08.009

Wei, G. W. 2010d. GRA method for multiple attribute decision making with incomplete weight information in intuitionistic fuzzy setting, Knowledge-Based Systems 23(3): 243-247.

http://dx.doi.org/10.1016/j.knosys.2010.01.003

Wei, G. W.; Lin, R.; Zhao, X. F.; Wang, H. J. 2010a. Models for multiple attribute group decision making with 2-tuple linguistic assessment information, International Journal of Computational Intelligence Systems 3(3): 315-324. http://dx.doi.org/10.1080/18756891.2010.9727702

Wei, G. W.; Zhao, X. F.; Lin, R. 2010b. Some induced aggregating operators with fuzzy number intuitionistic fuzzy information and their applications to group decision making, International Journal of Computational Intelligence Systems 3(1): 84-95.

$\mathrm{Xu}, \mathrm{Z}$. S. 2004a. A method based on linguistic aggregation operators for group decision making with linguistic preference relations, Information Sciences 166(1): 19-30.

http://dx.doi.org/10.1016/j.ins.2003.10.006

Xu, Z. S. 2004b. Uncertain linguistic aggregation operators based approach to multiple attribute group decision making under uncertain linguistic environment, Information Science 168: 171-184. http://dx.doi.org/10.1016/j.ins.2004.02.003

$\mathrm{Xu}, \mathrm{Z}$. S. 2004c. EOWA and EOWG operators for aggregating linguistic labels based on linguistic preference relations, International Journal of Uncertainty, Fuzziness and Knowledge-Based Systems 12: 791-810. http://dx.doi.org/10.1142/S0218488504003211

$\mathrm{Xu}, \mathrm{Z}$. S. 2005a. A method based on IA operator for multiple attribute group decision making with uncertain linguistic information, Lecture Notes in Artificial Intelligence 3613: 684-693. 
Xu, Z. S. 2005b. An approach to pure linguistic multiple attribute decision making under uncertainty, International Journal of Information Technology and Decision Making 4: 197-206. http://dx.doi.org/10.1142/S0219622005001465

$\mathrm{Xu}, \mathrm{Z}$. S. 2005c. Deviation measures of linguistic preference relations in group decision making, Omega 33: 249-254. http://dx.doi.org/10.1016/j.omega.2004.04.008

$\mathrm{Xu}, \mathrm{Z}$. S. 2006a. A note on linguistic hybrid arithmetic averaging operator in multiple attribute group decision making with linguistic information, Group Decision and Negotiation 15(6): 59-604. http://dx.doi.org/10.1007/s10726-005-9008-4

$\mathrm{Xu}, \mathrm{Z}$. S. 2006b. Induced uncertain linguistic OWA operators applied to group decision making, Information Fusion 7: 231-238. http://dx.doi.org/10.1016/j.inffus.2004.06.005

$\mathrm{Xu}, \mathrm{Z}$. S. 2006c. An approach based on the uncertain LOWG and induced uncertain LOWG operators to group decision making with uncertain multiplicative linguistic preference relations, Decision Support Systems 41: 488-499. http://dx.doi.org/10.1016/j.dss.2004.08.011

$\mathrm{Xu}, \mathrm{Z}$. S. 2006d. On generalized induced linguistic aggregation operators, International Journal of General Systems 35: 17-28. http://dx.doi.org/10.1080/03081070500422836

$\mathrm{Xu}, \mathrm{Z}$. S. 2007a. A method for multiple attribute decision making with incomplete weight information in linguistic setting, Knowledge-Based Systems 20(8): 719-725.

http://dx.doi.org/10.1016/j.knosys.2006.10.002

$\mathrm{Xu}$, Z. S. 2007b. Group decision making with triangular fuzzy linguistic variables, in H. Yin, et al. (Eds.). IDEAL 2007, LNCS 4881, Springer, 17-26.

$\mathrm{Xu}, \mathrm{Z}$. S. 2007c. An interactive procedure for linguistic multiple attribute decision making with incomplete weight information, Fuzzy Optimization and Decision Making 6: 17-27.

http://dx.doi.org/10.1007/s10700-006-0022-z

$\mathrm{Xu}, \mathrm{Z}$. S. 2008. Harmonic mean operators for aggregating linguistic information, in The 4th International Conference on Natural Computation, 25-27 August 2008, Jinan, China, 204-208.

$\mathrm{Xu}$, Z. S. 2009a. Fuzzy harmonic mean operators, International Journal of Intelligent Systems 24: 152-172. http://dx.doi.org/10.1002/int.20330

$\mathrm{Xu}, \mathrm{Z}$. S. 2009b. Correlated linguistic information aggregation, International Journal of Uncertainty, Fuzziness and Knowledge-Based Systems 17(5): 633-647.

http://dx.doi.org/10.1142/S0218488509006182

$\mathrm{Xu}, \mathrm{Z}$. S. 2010. Choquet integrals of weighted intuitionistic fuzzy information, Information Sciences 180: 726-736. http://dx.doi.org/10.1016/j.ins.2009.11.011

Yager, R. R. 1988. On ordered weighted averaging aggregation operators in multicriteria decision making, IEEE Transactions on Systems, Man, and Cybernetics 18: 183-190.

http://dx.doi.org/10.1109/21.87068

Yager, R. R. 2004. Generalized OWA aggregation operators, Fuzzy Optimization and Decision Making 3: 93-107. http://dx.doi.org/10.1023/B:FODM.0000013074.68765.97

Ye, J. 2009a. Multicriteria fuzzy decision-making method based on a novel accuracy function under interval-valued intuitionistic fuzzy environment, Expert Systems with Applications 36(3): 6899-6902. http://dx.doi.org/10.1016/j.eswa.2008.08.042

Ye, J. 2009b. Using an improved measure function of vague sets for multicriteria fuzzy decisionmaking, Expert Systems with Applications 37(6): 4706-4709.

http://dx.doi.org/10.1016/j.eswa.2009.11.084

Zhao, X. F.; Wei, G. W. 2009. Fuzzy linguistic hybrid geometric aggregation operator, Journal of Computational Information Systems 5(5): 1419-1424. 
Rui LIN is a lecturer in School of Economics and Management, Chongqing University of Arts and Sciences. He received the B.E. degree in management sciences and engineer from Chengdu University of Technology, China. He has worked for Department of Economics and Management, Chongqing University of Arts and Sciences, China as a lecturer since 2007.

Guiwu WEI has a MSc and a PhD degree in applied mathematics from SouthWest Petroleum University, Business Administration from school of Economics and Management at SouthWest Jiaotong University, China, respectively. He is a Professor in the School of Economics and Management at Chongqing University of Arts and Sciences. He has published more than 90 papers in journals, books and conference proceedings including journals such as Expert Systems with Applications, Applied Soft Computing, Knowledge and Information Systems, Knowledge-based Systems, International Journal of Uncertainty, Fuzziness and Knowledge-Based Systems, International Journal of Computational Intelligence Systems and Information: An International Journal. He has published 1 book. He has participated in several scientific committees and serves as a reviewer in a wide range of journals including Computers \& Industrial Engineering, International Journal of Information Technology and Decision Making, Knowledge-based Systems, Information Sciences, International Journal of Computational Intelligence Systems and European Journal of Operational Research. He is currently interested in Aggregation Operators, Decision Making and Computing with Words.

Hongjun WANG is a lecturer in School of Economics and Management, Chongqing University of Arts and Sciences. He received the B.E.and M.E. degree in management sciences and engineer from SouthWest Petroleum University, China. He has worked for Department of Economics and Management, Chongqing University of Arts and Sciences, China as a lecturer since 2006.

Xiaofei ZHAO is a lecturer in School of Economics and Management, Chongqing University of Arts and Sciences. He received the B.E. degree in applied mathematics from Southwest University, China. He has worked for Department of Economics and Management, Chongqing University of Arts and Sciences, China as a lecturer since 2008. 